\title{
Air embolism after peripheral IV contrast injection
}

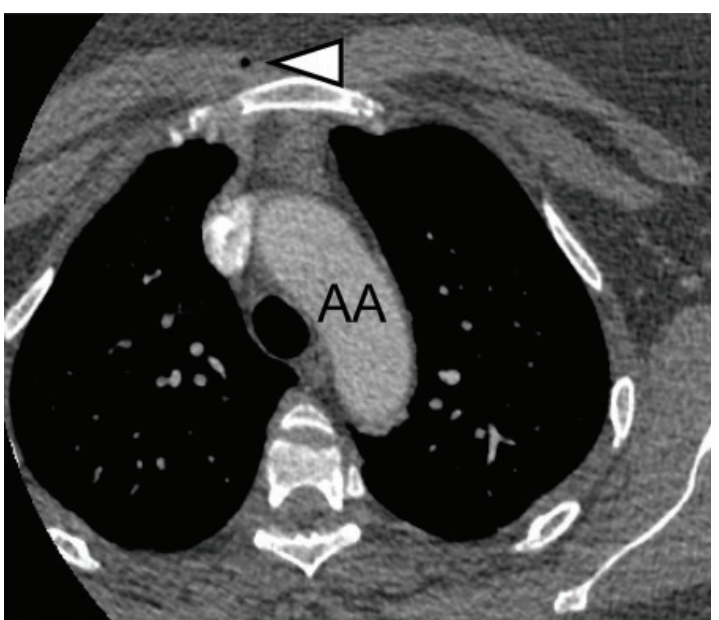

As little as

$0.5 \mathrm{~mL}$ of air in the coronary arteries

can cause

ventricular

fibrillation;

2 to $3 \mathrm{~mL}$ of air in the cerebral circulation can be fatal
Figure 1. Computed tomography showed an air bubble (arrowhead) in the peripheral vasculature of the anterior chest wall at the level of the aortic arch (AA).

A5-YEAR-OLD woman with hypertension $A$ presented with acute intermittent abdominal pain radiating to her back.

Her blood pressure was 139/88 mm Hg in the right upper extremity and $133 / 80 \mathrm{~mm} \mathrm{Hg}$ in the left upper extremity. Her heart rate was regular, and breath sounds were clear. She had mild abdominal tenderness in the umbilical and left lumbar regions.

Electrocardiography and laboratory testing, including troponins, showed no abnormalities. Computed tomography of the chest and abdomen with contrast enhancement showed no evidence of aortic dissection or pulmonary embolism. However, postcontrast images detected nonobstructing calculi in the left kidney and air emboli in the peripheral venous

The authors report no relevant financial relationships which, in the context of their contributions, could be perceived as a potential conflict of interest. doi:10.3949/ccjm.87a.20001

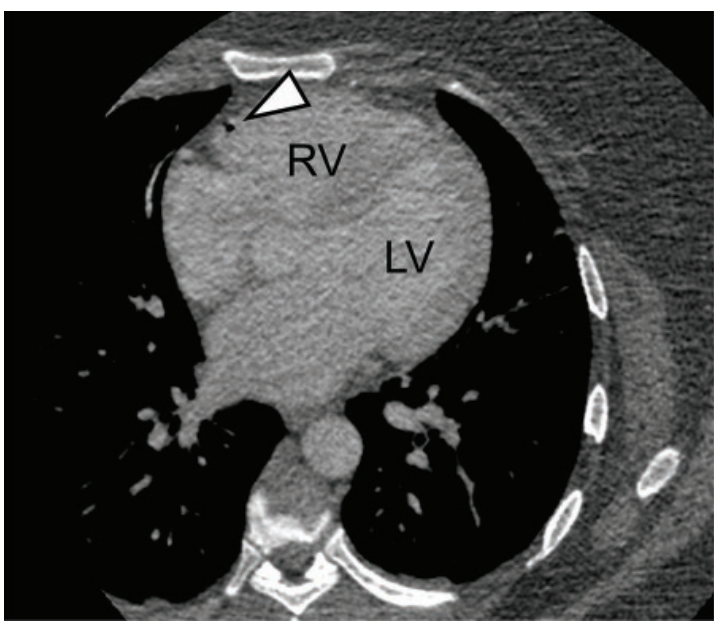

Figure 2. An air embolus (arrowhead) along the anterior wall of the right ventricle (RV).

circulation (Figure 1), right ventricle (Figure 2 ), and pulmonary trunk (Figure 3). The air emboli were thought to be most likely from peripheral intravenous catheter placement or manipulation for contrast injection.

The patient remained hemodynamically stable, with no new-onset chest pain, shortness of breath, or neurologic signs. She was placed in the Trendelenburg position, and high-flow oxygen supplementation was started. Transthoracic echocardiography showed normal left ventricular systolic function with a $66 \%$ ejection fraction, and normal right ventricle size and function with an estimated systolic pressure of $26 \mathrm{~mm} \mathrm{Hg}$. The patient was maintained on supplemental oxygen and was encouraged to lie in the left lateral decubitus position. Her abdominal pain was likely due to nephrolithiasis, which improved with hydration and analgesia, and she was discharged home. 


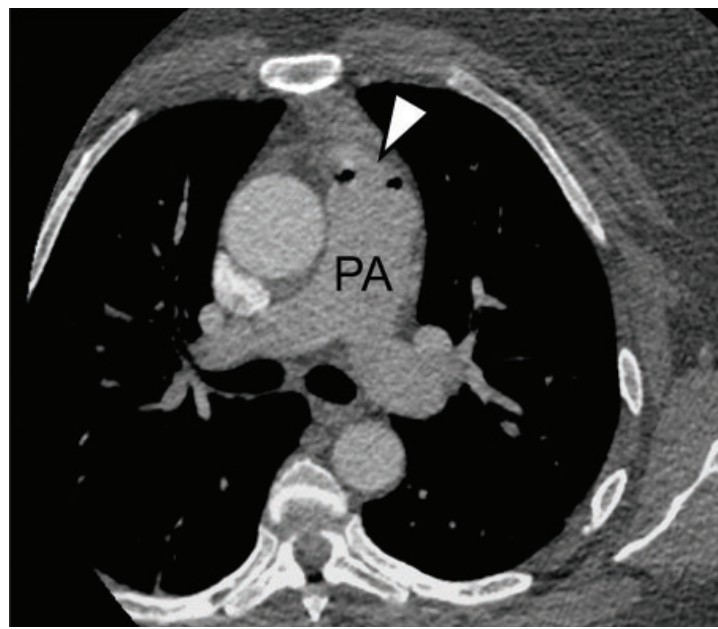

Figure 3. Multiple air emboli (arrowhead) in the proximal pulmonary artery (PA).

\section{CAUSES OF VENOUS AIR EMBOLISM}

Venous air embolism is a potentially catastrophic complication when it occludes blood flow to the pulmonary, cardiac, or cerebral circulation. It is most often associated with neurologic and otolaryngologic surgery, thoracic or abdominal trauma (including barotrauma from mechanical ventilation), and vascular interventions such as central and peripheral intravenous access. ${ }^{1}$ It can also occur when central venous pressure is low, such as when a catheter is inserted during inspiration, when the patient is upright, or if the patient is hypovolemic. ${ }^{1,2}$ Alternatively, incorrectly flushed lines or pressurized injection of fluids, such as contrast media, may force air into the vasculature. ${ }^{3,4}$

While venous air embolism is a known risk associated with intravenous access, many cases are asymptomatic and therefore go undetected, likely leading to an underestimation of the true incidence. ${ }^{4}$ A study of more than 11,000 central venous catheter insertions using optimal positioning and technique found an incidence of $0.13 \% .{ }^{1}$ A study of $677 \mathrm{pa}$ tients who underwent peripheral contrast injection observed an incidence of $11.7 \%{ }^{4}$

\section{MANAGEMENT}

Management of venous air embolism depends on the location and volume of the emboli. In most cases, the air is resorbed without complications. However, large emboli or those in patients with shunts that can result in paradoxical emboli may present with cardiopulmonary or neurologic symptoms. ${ }^{1,2}$ As little as $0.5 \mathrm{~mL}$ of air in the coronary arteries can cause ventricular fibrillation, and 2 to $3 \mathrm{~mL}$ of air in the cerebral circulation can be fatal. ${ }^{5}$

High-flow oxygen therapy helps maintain adequate oxygenation and promotes air resorption. ${ }^{1}$ Placing the patient in the Trendelenburg or left lateral decubitus position (Durant maneuver) encourages air emboli to migrate away from the right ventricular outflow tract and toward the right atrium, avoiding possible cardiopulmonary collapse. ${ }^{1,6}$ If available, hyperbaric oxygen therapy is the definitive treatment for severe cases and has been shown to improve recovery when administered early. ${ }^{1}$

\section{MINIMIZING THE RISK}

The risk of venous air embolism can be minimized when inserting or removing a central venous catheter by placing the patient in the supine or Trendelenburg position and ensuring that the patient is hydrated. Catheter insertion should be avoided during inspiration, and removal should be performed while the patient performs a Valsalva maneuver or during exhalation.

Before contrast injection, the catheter lumen should be flushed, and components such as stopcocks and Luer locks should be inspected and secured.

Incidentally detected intracardiac air emboli rarely cause hemodynamic compromise. However, as in our patient, appropriate management is straightforward and effective in preventing potentially life-threatening cardiopulmonary and neurologic complications.

\author{
Incorrectly \\ flushed lines \\ or pressurized \\ injection of \\ fluids, such as \\ contrast media, \\ may force \\ air into \\ the vasculature
}

.




\section{AIR EMBOLISM}

REFERENCES

1. McCarthy CJ, Behravesh S, Naidu SG, Oklu R. Air embolism: practical tips for prevention and treatment. J Clin Med 2016; 5(11):93. doi: $10.3390 / \mathrm{jm} 5110093$

2. Gordy S, Rowell S. Vascular air embolism. Int J Crit IIIn Inj Sci 2013; 3(1):73-76. doi:10.4103/2229-5151.109428

3. Ie SR, Rozans MH, Szerlip HM. Air embolism after intravenous injection of contrast material. South Med J 1999; 92(9):930-933. doi:10.1097/00007611-199909000-00019

4. Groell R, Schaffler GJ, Rienmueller R, Kern R. Vascular air embolism: location, frequency, and cause on electron-beam CT studies of the chest.
Radiology 1997; 202(2):459-462. doi:10.1148/radiology.202.2.9015074

5. Ho AM. Is emergency thoracotomy always the most appropriate immediate intervention for systemic air embolism after lung trauma? Chest 1999; 116(1):234-237. doi:10.1378/chest.116.1.234

6. Ericsson JA, Gottlieb JD, Sweet RB. Closed-chest cardiac massage in the treatment of venous air embolism. N Engl J Med 1964; 270:1353-1354. doi:10.1056/NEJM196406182702507

Address: Karthik Gnanapandithan, MD, MS, FHM, Yale School of Medicine, 333 Cedar St., New Haven, CT 06510;

karthik.gnanapandithan@ynhh.org 